\title{
Plataforma virtual enfocada a tecnologías del aprendizaje y conocimiento a nivel preescolar
}

\author{
Hugo Montes de Oca-Martínez \\ hugo.montes@tesh.edu.mx \\ Licenciatura en Informática \\ Tecnológico de Estudios Superiores de Huixquilucan, México.

\section{María Teresa Cruz-Gordillo} \\ maria.cruz@tesh.edu.mx \\ Maestría en Dirección de Tecnologías de la Información \\ Tecnológico de Estudios Superiores de Huixquilucan, México.
}

Recepción:15 de julio del 2020

Aprobación:15 de septiembre del 2020

Publicación: 06 de junio del 2021

\section{Resumen}

La enseñanza académica por medio de la integración tecnológica para niños de preescolar se ha vuelto una herramienta o instrumento de aprendizaje muy eficaz ya que despierta habilidades del conocimiento específicos y esto conlleva a fortalecer los currículos educativos de las instituciones.

El presente trabajo de investigación muestra el desarrollo de una Plataforma Virtual Enfocada a Tecnologías del Aprendizaje y Conocimiento a Nivel Preescolar, para mejorar la calidad educativa de los niños del Instituto pedagógico Jean Piaget perteneciente al municipio de Huixquilucan de Degollado, Estado de México. Dicho proyecto consiste en el diseñar una aplicación de realidad aumentada que sirve como instrumento educativo para el proceso del "saber", de los estudiantes.

Para el diseño de cada elemento animado en 3D se manejaron diferentes programas, así como la utilización de la metodología cualitativa ya que permite realizar un estudio a la población por medio de la observación, con la plataforma virtual se pretende fortalecer la enseñanza del niño de preescolar de manera más significativa.

Palabras claves: Virtual, Enseñanza, Aprendizaje, Metodología. 


\title{
Virtual platform focused on learning technologies and knowledge at the preschool level
}

\begin{abstract}
Academic teaching through technological integration for preschool children has become a very effective learning tool or instrument since it awakens specific knowledge skills and this leads to strengthening the educational curricula of institutions.

This research work shows the development of a Virtual Platform Focused on Learning Technologies and Knowledge at the Preschool Level, to improve the educational quality of children from the Jean Piaget Pedagogical Institute belonging to the municipality of Huixquilucan de Degollado, State of Mexico. This project consists of designing an augmented reality application that serves as an educational instrument for the process of "knowing" of the students.

For the design of each 3D animated element, different programs were managed, as well as the use of the qualitative methodology since it allows a study of the population through observation, with the virtual platform it is intended to strengthen the teaching of preschool children more significantly.
\end{abstract}

Key words: Virtual, Teaching, Learning, Methodology

\section{Introducción}

Las aplicaciones móviles se han presentado en estos últimos años como un instrumento o herramienta de nuestra vida cotidiana ya que nos permiten realizar múltiples tareas en cualquier tiempo y espacio.

Según el Autor Muñoz, (2019)'La realidad aumentada (RA) es el término que se usa para describir al conjunto de tecnologías que permiten que un usuario visualice parte del mundo real a través de un dispositivo tecnológico con información gráfica añadida por éste dispositivo”.

El presente documento muestra el desarrollo de una aplicación denominada: "Plataforma Virtual Enfocada a Tecnologías del Aprendizaje y conocimiento a Nivel Preescolar”, el cual fue contribución de un proyecto de investigación de la carrera de Ingeniería en Sistemas Computacionales adscrita al Tecnológico de Estudios Superiores de Huixquilucan, con el objetivo de aportar a los pedagogos del Instituto Jean Piaget una aplicación de realidad aumentada para el 
aprendizaje en distintas disciplinas del conocimiento. Cabe resaltar que para la construcción de la plataforma se realizaron una serie de actividades para la compresión de análisis, desarrollo e implementación.

En el contenido del documento se especifica la sustentación de la investigación para comprender las bases del proyecto, así como las herramientas empleadas para el desarrollo y diseño de la Plataforma Virtual.

\section{Contexto del Proyecto.}

Las instituciones pedagógicas tienen que irse adecuando a los cambios sociales y tecnológicos para alcanzar la calidad formativa de sus estudiantes, pero esto conlleva a hacer modificaciones en el diseño de la currícula educativa, así como las formas de enseñanza-aprendizaje mediante las Tecnologías de Información y Comunicación, debido a que los estudiantes de preescolar inclinan más sus intereses a la era digital.

El Programa para la Evaluación Internacional de Alumnos (PISA), es un proyecto que entre otras actividades se encarga de medir el desempeño de los estudiantes en México, dicho programa menciona que falta innovación en las aulas de clases particularmente en el nivel preescolar es débil y claramente disfuncional ya que hace falta emplear las TIC'S para la enseñanza de los niños. Con fundamento de lo anterior el Instituto Pedagógico Jean Piaget, preocupado por la enseñanza de sus estudiantes se puso a disposición con los docentes de tiempo completo de la carrera de Ingeniería en Sistemas Computacionales, para el desarrollo de una plataforma virtual enfocada al aprendizaje, que servirá como auge en los estudiantes de preescolar e innovando de forma más significativa y dinámica la enseñanza.

El articulo muestra el desarrollo de una plataforma enfocada a las tecnologías del aprendizaje y conocimiento para niños de nivel preescolar, dicho proyecto está enfocado a una línea de investigación denominada "Sistemas, Bases de Datos y Plataformas Computacionales", teniendo como finalidad primordial brindar a los pedagogos un instrumento interactivo para el desarrollo de habilidades y enseñanza cognoscitiva en las distintas disciplinas del conocimiento como son los números, vocales y abecedario entre otras, con el objetivo de alcanzar en los niños de nivel preescolar un aprendizaje más significativo y dinámico. 


\section{Materiales y Métodos}

Los programas y procedimientos que se aplicaron para el diseño del proyecto de investigación son las siguientes:

\section{Realidad Aumentada con Vuforia}

Vuforia es un SDK que permite construir aplicaciones basadas en la Realidad Aumentada; una aplicación desarrollada con Vuforia utiliza la pantalla del dispositivo como un "lente mágico" en donde se entrelazan elementos del mundo real con elementos virtuales (como letras, imágenes, etc.). Al igual que con Wikitude, la cámara muestra a través de la pantalla del dispositivo, vistas del mundo real, combinados con objetos virtuales como: modelos, bloque de textos, imágenes, etc.

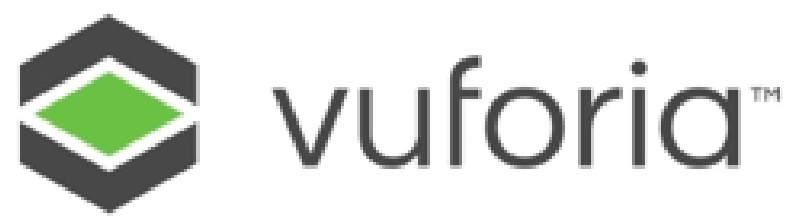

Figura 1 Logo de Vuforia

Fuente: (Jiménez, 2019). Jiménez, D. (24 de Septiembre de 2019). Vuforia y el deployment de aplicaciones. Obtenido de http://niixer.com/2019/09/24/vuforia-y-el-deployment-de-aplicaciones/

\section{Arquitectura de Vuforia}

Una aplicación desarrollada con Vuforia está compuesta de los siguientes elementos:

Cámara: La cámara asegura que la imagen sea captada y procesada por el Tracker.

Base de datos: La base de datos del dispositivo es creada utilizando el Target Manage; ya sea la base de datos local o la base de datos en la nube, almacena una colección de Targets para ser reconocidos por el Tracker.

Target: Son utilizadas por el rastreador (Tracker) para reconocer un objeto del mundo real; los Targets pueden ser de diferentes tipos; entre los principales tenemos:

Tracker: Analiza la imagen de la cámara y detecta objetos del mundo real a través de los frame de la cámara con el fin de encontrar coincidencias en la base de datos. (Motor Vuforia compatible con Unity, 2020) 


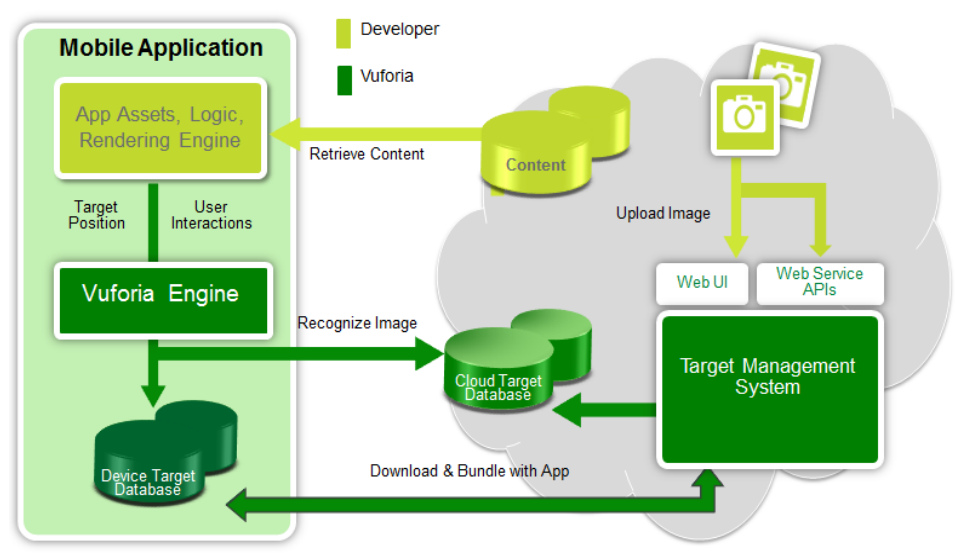

Figura 2 Arquitectura de Vuforia

\section{Unity}

Unity es una herramienta de desarrollo de videojuegos creada por la empresa Unity Technologies. En la página web de Unity, en la sección Made with Unity, podemos ver que este software se ha utilizado para crear multitud de juegos conocidos y otros no tan conocidos. También se ha utilizado para crear experiencias de Realidad Virtual interactivas e incluso miniseries, como "Baymax Dreams", producida por Disney junto con Unity, donde se ha utilizado el editor para procesar y previsualizar en tiempo real todos los capítulos de la miniserie.

\section{\&unity}

Figura 3 Logo de Unity

\section{Blender}

Blender es la suite de creación 3D gratuita y de código abierto. Admite la totalidad de la canalización 3D: modelado, aparejo, animación, simulación, renderizado, composición y seguimiento de movimiento, edición de video y canalización de animación 2D.

Blender es un proyecto público, realizado por cientos de personas de todo el mundo; por estudios y artistas individuales, profesionales y aficionados, científicos, estudiantes, expertos en efectos visuales, animadores, artistas de juegos, modders, y la lista continúa. (Vazquez, Siddi, 
McGrath, Sharybin, \& Roosendaal, 2005)

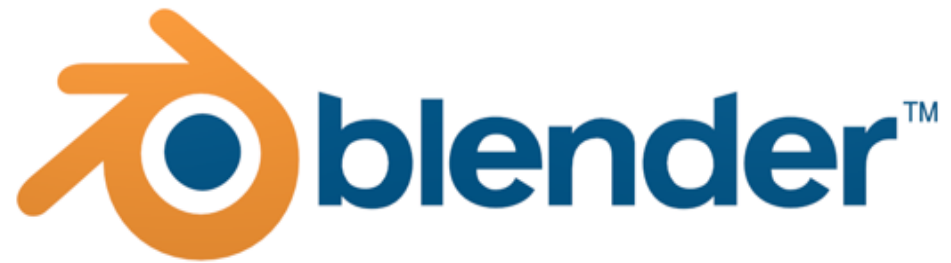

Figura 4 Logo de Blender

\section{Android SDK}

El SDK (Software Development Kit) de Android, incluye un conjunto de herramientas de desarrollo. Comprende un depurador de código, biblioteca, un simulador de teléfono basado en QEMU, documentación, ejemplos de código y tutoriales. Las plataformas de desarrollo soportadas incluyen GNU/Linux, Mac OS X 10.5.8 o posterior, y Windows XP o posterior. La plataforma integral de desarrollo (IDE, Integrated Development Environment) soportada oficialmente es Android Studio junto con el complemento ADT (Android Development Tools plugin). Además, los programadores pueden usar un editor de texto para escribir ficheros Java y XML y utilizar comandos en un terminal (se necesitan los paquetes JDK, Java Development Kit y Apache Ant) para crear y depurar aplicaciones, así como controlar dispositivos Android que estén conectados ( es decir, reiniciarlos, instalar aplicaciones en remoto, etc.). (Google, 2019).

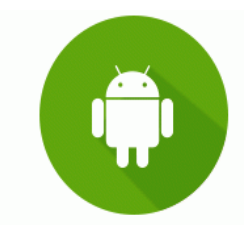

\section{Android SDK}

Figura 5 Logo de Android SDK

\section{JDK}

El JDK es el Java Development Kit, que traducido al español es, Herramientas de desarrollo para Java, aquí nos encontraremos con el compilador javac que es el encargado de convertir nuestro código fuente (.java) en bytecode (.class), el cual posteriormente sera interpretado y ejecutado con la JVM, Java Virtual Machine por sus siglas en inglés, que nuevamente al español es La Máquina Virtual de Java, también dentro de estas herramientas encontramos los siguientes 
programas, javadoc(encargado de generar la documentación de nuestro código), el jvisualvm (muestra información a detalle sobre las aplicaciones que están corriendo actualmente en la JVM), entre muchas otras. (Perales, 2015)

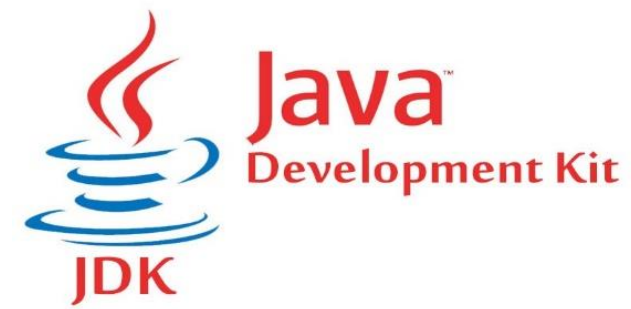

Figura 6 JDK de Java 


\section{Desarrollo del Proyecto}

Cronograma de Actividades

\begin{tabular}{|c|c|c|c|c|c|c|c|c|c|}
\hline \multirow{3}{*}{ No. } & \multirow{3}{*}{ Actividades por cada etapa del proyecto } & Prog. & \multirow{3}{*}{ Mar. } & \multirow{3}{*}{ Abr. } & \multirow{3}{*}{ May } & \multirow{3}{*}{ Jun. } & \multirow{3}{*}{ Jul. } & \multirow{3}{*}{ Ago. } & \multirow{3}{*}{ Sept. } \\
\hline & & $\mathbf{P}$ & & & & & & & \\
\hline & & $\mathbf{R}$ & & & & & & & \\
\hline \multicolumn{10}{|c|}{ Etapa 1 Plan inicial } \\
\hline 1 & Definición del nrovecto & $\mathrm{P}$ & & & & & & & \\
\hline 1 & Derinicion der proyecto & $\mathrm{R}$ & & & & & & & \\
\hline 2 & \multirow[t]{2}{*}{ Realizar benchmarking } & $\begin{array}{ll}P \\
R\end{array}$ & & & & & & & \\
\hline & & & & & & & & & \\
\hline \multicolumn{10}{|c|}{ Etapa 2 Planificación } \\
\hline 1 & Planificación para tomar acciones & $P$ & & & & & & & \\
\hline \multirow[b]{2}{*}{2} & \multirow{2}{*}{ Objetivos y planificación para lógralos } & $\frac{R}{P}$ & & & & & & & \\
\hline & & $R$ & & & & & & & \\
\hline & & & & & & & & & \\
\hline \multicolumn{10}{|c|}{ Etapa 3 Requisitos } \\
\hline 1 & Determinar las necesidades del usuario & $P$ & & & & & & & \\
\hline \multirow{2}{*}{2} & \multirow{2}{*}{ Elaboración de Encuestas } & $\frac{n}{p}$ & & & & & & & \\
\hline & & $\mathrm{R}$ & & & & & & & \\
\hline \multirow[t]{2}{*}{3} & Análisis de requerimientos de Información & $\frac{P}{R}$ & & & & & & & \\
\hline & & & & & & & & & \\
\hline \multicolumn{10}{|c|}{ Etapa 4 Análisis y diseño } \\
\hline \multirow{2}{*}{1} & \multirow{2}{*}{ Desarrollo de Interfaz } & $\mathrm{P}$ & & & & & & & \\
\hline & & $\mathrm{R}$ & & & & & & & \\
\hline \multirow[t]{2}{*}{2} & \multirow[t]{2}{*}{ Diseño Inicial } & $\frac{P}{R}$ & & & & & & & \\
\hline & & & & & & & & & \\
\hline \multicolumn{10}{|c|}{ Etapa 5 Implementación } \\
\hline 1 & Gestión de cambio & $P$ & & & & & & & \\
\hline & & $\mathrm{R}$ & & & & & & & \\
\hline Etapa 6 P & & & & & & & & & \\
\hline 1 & Evaluación de Criterios de salida & $P$ & & & & & & & \\
\hline & & $\frac{R}{P}$ & & & & & & & \\
\hline 2 & Evaluar resultados & $\mathrm{R}$ & & & & & & & \\
\hline Document & y difusión del proyecto & & & & & & & & \\
\hline 1 & Documentación de las etapas del proyecto & $\frac{P}{R}$ & & & & & & & \\
\hline
\end{tabular}

Tabla 7 Cronograma de Actividades

Revista RedCA, Vol. 4, Núm. 10 (junio-septiembre 2021) 


\section{Descripción de las Actividades}

\section{Etapa 1 Plan Inicial}

Definición del Proyecto: Se estableció un cronograma de actividades para el desarrollo y diseño de la Plataforma Virtual Enfocada a Tecnologías del Aprendizaje y Conocimiento a Nivel Preescolar.

Se definió el objetivo y los alcances sobre el proyecto de investigación.

Realizar Benchmarking: Recopilación de información sobre la existencia de proyectos similares a la Plataforma Virtual, para analizar o buscar las áreas de oportunidad para el aprendizaje significativo de los niños de preescolar.

La recopilación de datos del benchmarking (véase anexo 1) los resultados:

\section{Etapa 2 Planificación}

Planificación para Tomar Acciones: Se realizó una serie de acciones para planificar las tareas asociadas a cada objetivo para la eficiencia terminal del diseño y desarrollo del proyecto de investigación.

Objetivos y Planificación para Lógralos: Se establecieron las funciones y procesos necesarios para la identificación del proyecto para conocer las condiciones para su estructuración de cada modelo.

\section{Etapa 3 Requisitos}

Determinar las Necesidades del Usuario: En el Instituto Pedagógico Jean Piaget se realizó una reunión con los directivos y pedagogos para identificar el objetivo claro del proyecto así como los requisitos de validación y verificación para la eficiencia terminal de la plataforma virtual.

Elaboración de Encuestas: Se diseñó un cuestionario para conocer las necesidades y el contexto de la enseñanza de los pedagogos hacia los niños, dichos datos fueron analizados y graficados para conocer el impacto y buscar áreas de oportunidad. 
Resultados más significativos del cuestionario de investigación de enseñanza-aprendizaje ¿Cuál es el mayor reto que se enfrenta al impartir un nuevo tema?
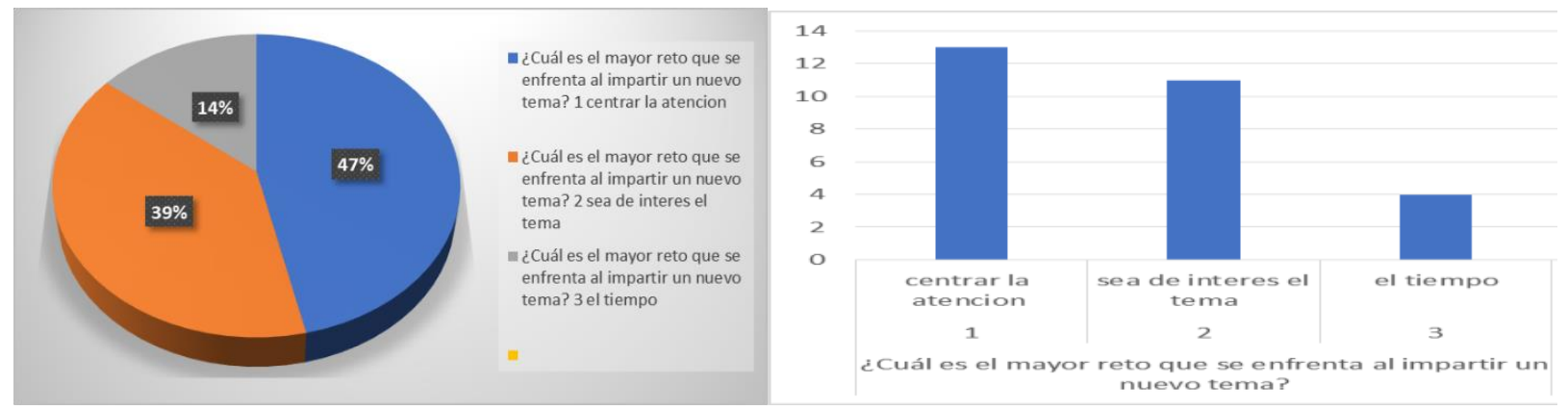

Figura 8 Resultado del reto

¿Cuál es su principal método de aprendizaje de los alumnos?
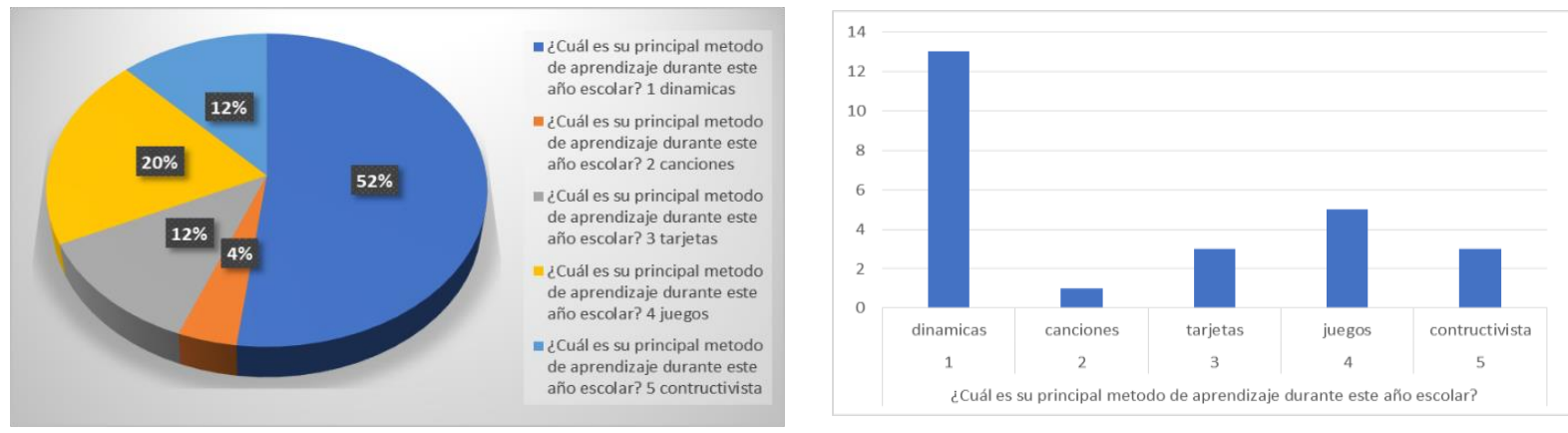

Figura 9 Resultado de método de aprendizaje

¿Utiliza alguna herramienta tecnológica como aplicaciones móviles para el aprendizaje de sus alumnos?
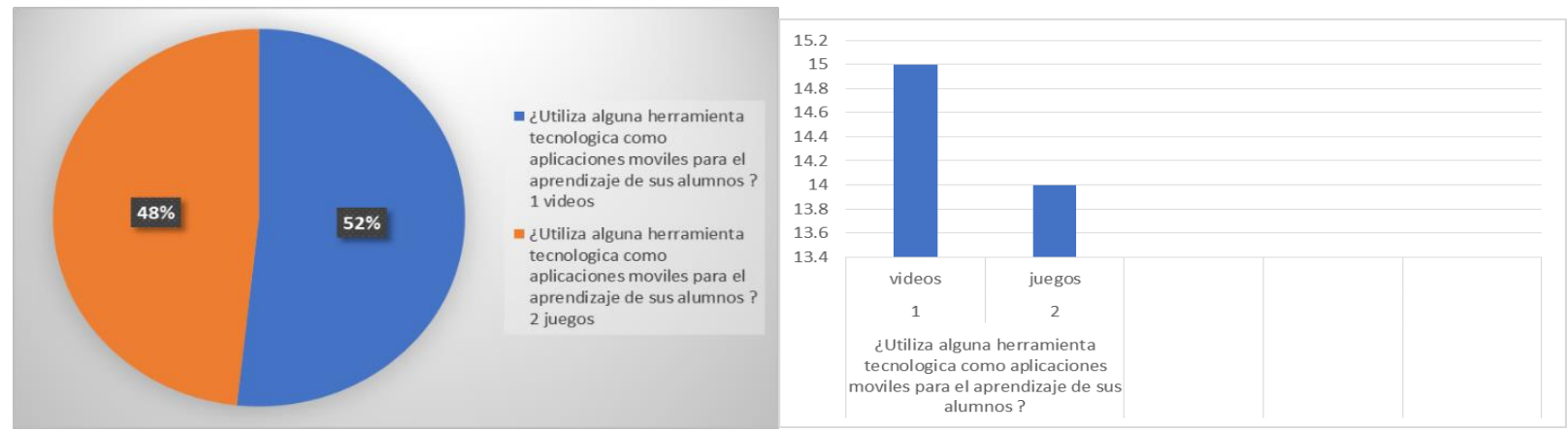
Figura 10 Resultado de utilizar tecnologías

Análisis de Requerimientos de Información: Se identificaron las áreas de conocimiento de gran importancia para el niño de preescolar.

\section{Análisis y Diseño}

Desarrollo de Interfaz: Se diseñó la base de datos para la identificación de cada objeto así como la configuración de cada paquete y librería para el funcionamiento correcto del proyecto.

Diseño Inicial: Se realizaron todos los modelos para la identificación y reconocimiento de las tarjetas.

En la figura 11 se presenta un fragmento de código para el movimiento y rotación de los elementos en $3 \mathrm{D}$.

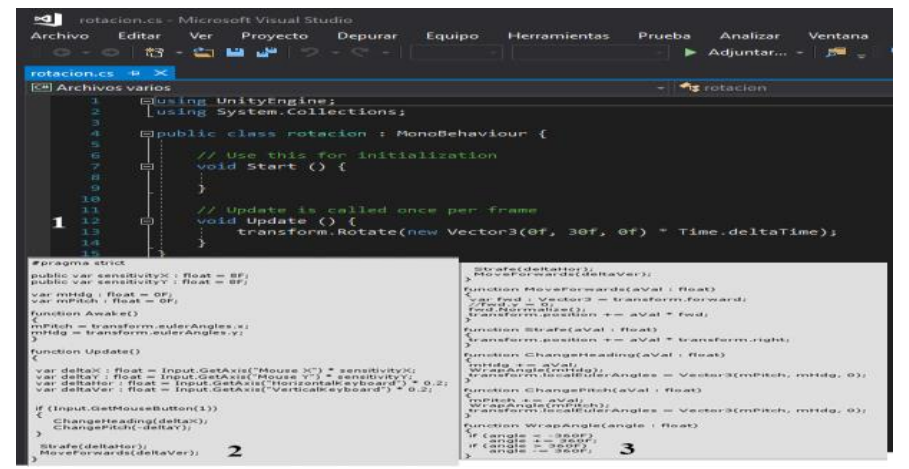

Figura 11 Programación en C\#

\section{Etapa 5 Implementación}

Gestión de Cambio: Se realizó una prueba piloto para ver la viabilidad y el funcionamiento correcto de la plataforma virtual.

\section{Etapa 6 Prueba}

Evaluación de Criterios de Salida: Se identificó que la versión de Android que fuera compatible con todos los dispositivos.

Evaluar Resultados: Se implementó la Plataforma Virtual Enfocada a Tecnologías del Aprendizaje y Conocimiento a Nivel Preescolar en varios dispositivos Android para identificar que las versiones no causaran ningún problema al ejecutarse y que funcione de manera ágil. Se presentó el proyecto con los directivos, pedagogos, padres de familia y los alumnos para que interactuaran con el instrumento de aprendizaje y evaluar su viabilidad. 
En la figura12 se ilustra la evidencia fotográfica de las pruebas realizadas en el Instituto Pedagógico Jean Piaget.

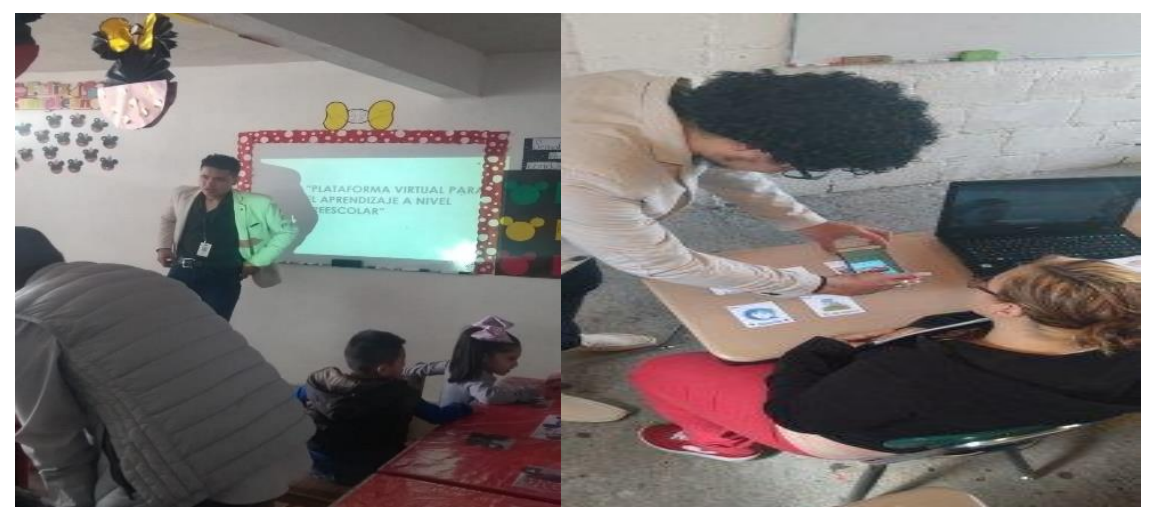

Figura 12 Prueba de la Aplicación

\section{Documentación y Difusión del Proyecto}

Documentación de las Etapas del Proyecto: En todo el proceso del proyecto se documentó cada etapa para el desarrollo eficiente de la plataforma así como la sustentación de los requisitos.

\section{Resultados de la Aplicación}

Se diseñó e implementó la Plataforma Virtual Enfocada a Tecnologías del Aprendizaje y Conocimiento a Nivel Preescolar influyendo significativamente en el ámbito educativo y social.

Diseño de la vocales en 3D.

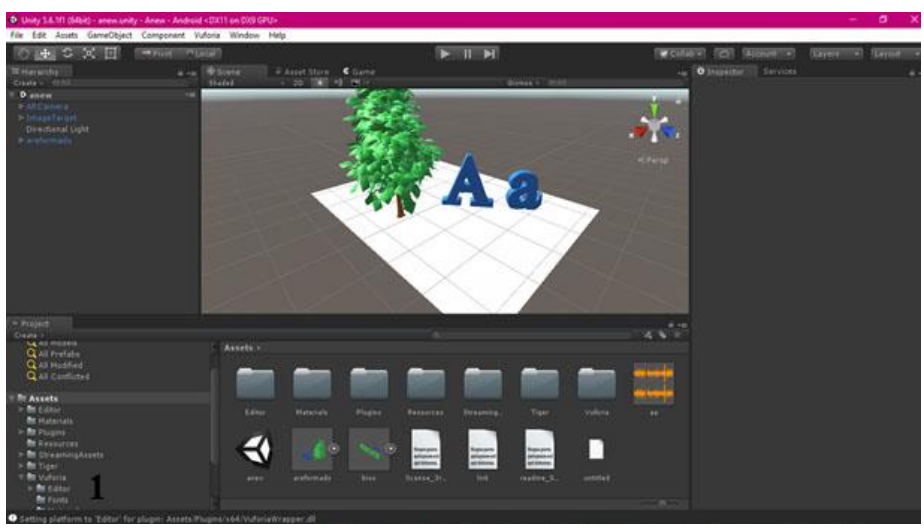

Figura 13 Diseño de Vocales 
Reconocimiento Webcam para cada objeto animado.
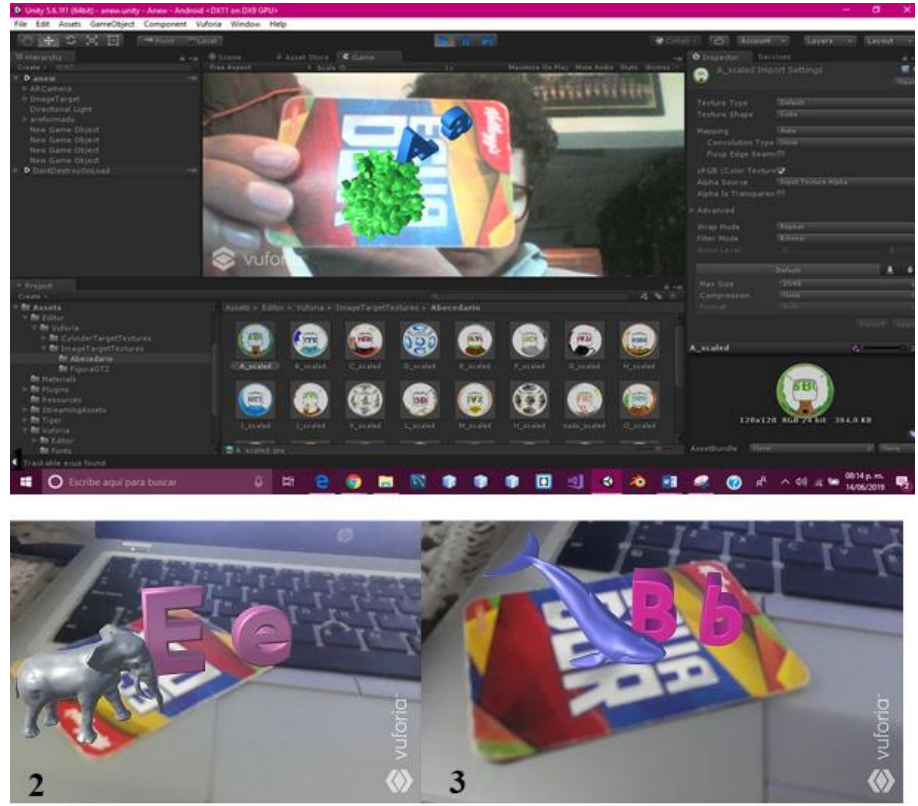

Figura 14 Reconocimiento Webcam

\section{Conclusiones}

Se implementó la plataforma virtual al Instituto Pedagógico Jean Piaget, beneficiando a 21 pedagogos y a 150 alumnos contribuyendo el proyecto de manera significativa para la enseñanzaaprendizaje de los niños de preescolar ya que permite interactuar dinámicamente en el reconocimiento de imágenes de 3D.

En este proyecto se manifestaron las necesidades reales de los pedagogos que trabajan día a día dentro del aula escolar, ya que es muy complicado atraer la atención de los niños de preescolar, por ende la plataforma contribuye con diferentes escenarios para aprendizaje significativo.

La Plataforma Virtual Enfocada a Tecnologías del Aprendizaje y Conocimiento a Nivel Preescolar se recomienda para su óptimo funcionamiento tener un dispositivo con versión de Android 4.0 o mayor. 


\section{Referencias}

(s.f.).

2.0, R. C. (16 de 10 de 2018). Camara del comercio de Bogota. Obtenido de Camara del comercio de Bogota: https://www.ccb.org.co/Sala-de-prensa/Noticias-EspecializacionInteligente/2017/Proyecto-de-realidad-virtual-y-realidad-aumentada-fue-ganador-en-laconvocatoria-de-Innpulsa-Reto-Cluster-2.0

Arreguin Gonzalez, J. F. (16 de 10 de 2018). REPOSITORIO INSTITUTACIONAL. Obtenido de REPOSITORIO INSTITUTACIONAL: http://ri.uaemex.mx/handle/20.500.11799/59266

Ayala Lliquín, E. M. (16 de 10 de 2018). Repositorio Institucional de la Escuela Superior Politécnica de Chimborazo. Obtenido de Repositorio Institucional de la Escuela Superior Politécnica de Chimborazo: Tesis Ingeniero en Sistemas Informáticos http://dspace.espoch.edu.ec/handle/123456789/6739

Colin, O. (diciembre de 2017). Google Maps. Obtenido de https://www.google.com/maps/uv?hl=es419\&pb=!1s0x85d20678d0b1eae1:0x967f631a2afe4111!3m1!7e115!4s/maps/place/Instit uto\%2BPedag\%25C3\%25B3gico\%2BJean\%2BPiaget/@19.4208039,99.3292154,3a,75y,49.93h,90t/data\%3D*213m4*211e1*213m2*211seDZZlwsP2hPNIFr $\mathrm{BGn} 2 \mathrm{PhA} * 212$

Cruz, A. (2014). Realidad Aumentada con Vuforia. Obtenido de https://www.desarrollolibre.net/blog/android/realidad-aumentada-convuforia\#.XpFUqMgzbIU

Erosa , D. (10 de junio de 2019). https://openwebinars.net/blog/que-es-unity/. Obtenido de Openwebinars: https://openwebinars.net/blog/que-es-unity/

Estela. (julio de 2014). Realidad Aumentada en $I+D+i$. Obtenido de http://investigacionrealidadaumentada.blogspot.com/2014/07/tecnicas-actuales-ensistemas-de.html

Fe, C. S. (16 de 10 de 2018). Ambiente de Realidad Aumentada Interactivo. Obtenido de Ambiente de Realidad Aumentada Interactivo: https://repositorio.itesm.mx/bitstream/handle/11285/614635/NOVUS_PaperAnatomia_Al encastre.pdf? sequence $=1$ \&isAllowed $=\mathrm{y}$

Garassini, M. E., \& Valery, C. P. (2004). Experiencias de uso de las TICs en la Educación 
Preescolar en Venezuela. ANALES dela Universidad Metropolitana, 225.

Google. (18 de julio de 2019). Wikipedia a enciclopedia libre. Obtenido de https://es.wikipedia.org/wiki/Android_SDK

Gutiérrez, Ó. (8 de Marzo de 2019). Apple lanzará sus gafas de realidad aumentada en 2020: Ming-Chi Kuo. Recuperado el 12 de Marzo de 2020, de https://www.cnet.com/es/noticias/apple-gafas-realidad-aumentada-2020/

Jiménez, D. (24 de Septiembre de 2019). Vuforia y el deployment de aplicaciones. Obtenido de http://niixer.com/2019/09/24/vuforia-y-el-deployment-de-aplicaciones/

Lizandra, D. M. (16 de 10 de 2018). Desarrollo de un sistema de. Obtenido de Desarrollo de un sistema de: https://riunet.upv.es/bitstream/handle/10251/8597/PFC\%20\%20Desarrollo\%20de\%20un\%20sistema\%20de\%20Realidad\%20Aumentada\%20en\%20 dispositivos\%20m\%C3\%B3viles.pdf

Motor Vuforia compatible con Unity. (28 de enero de 2020). Obtenido de https://developer.vuforia.com/

Muñoz, J. L. (2019). GeoGebra. Obtenido de https://www.geogebra.org/m/qbd7q6rr

Olmedo, M. E. (16 de 10 de 2018). INGLESVII_HEIDI_ZAMORA_NAVA_CORRECCIONES_15. Obtenido de INGLESVII_HEIDI_ZAMORA_NAVA_CORRECCIONES_15: http://repository.uaeh.edu.mx/bitstream/bitstream/handle/123456789/14792/INGLESVII_ HEIDI_ZAMORA_NAVA.pdf;sequence=2

Perales, I. (28 de Septiembre de 2015). Israel Perales Ingeniero en Tecnología y Software. Obtenido de https://www.ingenieroperales.com/que-es-el-jdk-y-el-jre-java/

Rivero, E. (16 de 10 de 2018). UNOCERO. Obtenido de UNOCERO: https://www.unocero.com/ciencia/el-ipn-simula-el-sistema-solar-con-realidad-virtual/

Vazquez, P., Siddi, F., McGrath, D., Sharybin, S., \& Roosendaal, T. (2005). Blender. Obtenido de https://www.blender.org/about/website/

Villalba, A. B. (16 de 10 de 2018). https://medium.com/@alvarobonilla/ispeak-vr-119890df4a3c. Obtenido de de https://medium.com/@alvarobonilla/ispeak-vr-119890df4a3c: https://medium.com/@alvarobonilla/ispeak-vr-119890df4a3c

Villalba, A. B. (16 de 10 de 2018). iSpeak VR. Obtenido de iSpeak VR: https://www.nacion.com/tecnologia/moviles/aplicacion-ensena-idiomas-con-ayuda-derealidad-virtual/ASP5HHNZG NFBLODGU6UEUCRFZU/story/ 


\section{Anexos}

\section{Anexo 1 Benchmarking}

Recopilación de información de proyectos direccionados a Plataforma Virtual Enfocada a Tecnologías del Aprendizaje y Conocimiento a Nivel Preescolar.

\section{VIRTUALIZACIÒN REALIDAD AUMENTADA}

\begin{tabular}{|c|c|c|}
\hline \multicolumn{3}{|c|}{ IPN } \\
\hline $\begin{array}{c}\text { NOMBRE DE LA } \\
\text { ESCUELA: }\end{array}$ & $\begin{array}{c}\text { PROYECTO } \\
\text { PRESENTADO }\end{array}$ & $\begin{array}{c}\text { AÑO EN QUE SE } \\
\text { PUBLICO }\end{array}$ \\
\hline $\begin{array}{l}\text { Universidad politécnica de } \\
\text { valencia escuela técnica } \\
\text { superior de ingeniería } \\
\text { informática }\end{array}$ & $\begin{array}{l}\text { Desarrollo de un sistema de } \\
\text { Realidad Aumentada en } \\
\text { dispositivos móviles }\end{array}$ & VALENCIA, 2010 \\
\hline \multicolumn{3}{|c|}{$\begin{array}{l}\text { Extraído: } \\
\text { itstream/handle/10251/8597/PFC\%20- } \\
\text { sistema\%20de\%20Realidad\%20Aumentada\%20en } \\
\text { tivos\%20m\%C3\%B3viles.pdf }\end{array}$} \\
\hline $\begin{array}{r}\text { Uno de los objetivos princi } \\
\text { sistema de Realidad Aume } \\
\text { ayuda al }\end{array}$ & $\begin{array}{l}\text { Descripción: } \\
\text { es de este proyecto es la impl } \\
\text { da (en adelante RA) en un di } \\
\text { amiento de la fobia a animale }\end{array}$ & $\begin{array}{l}\text { ación y validación de un } \\
\text { ivo móvil actual para la } \\
\text { ueños. }\end{array}$ \\
\hline
\end{tabular}




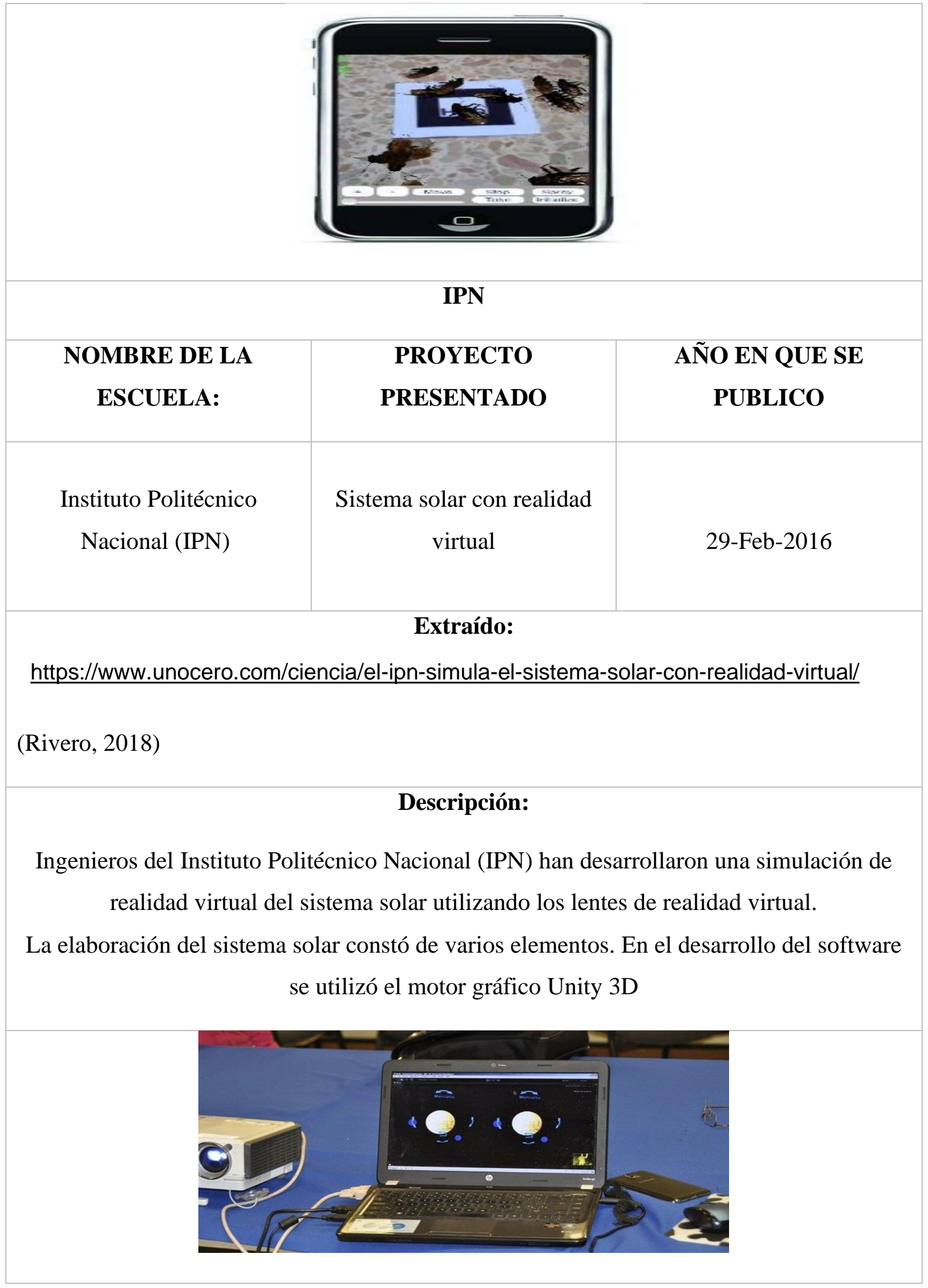




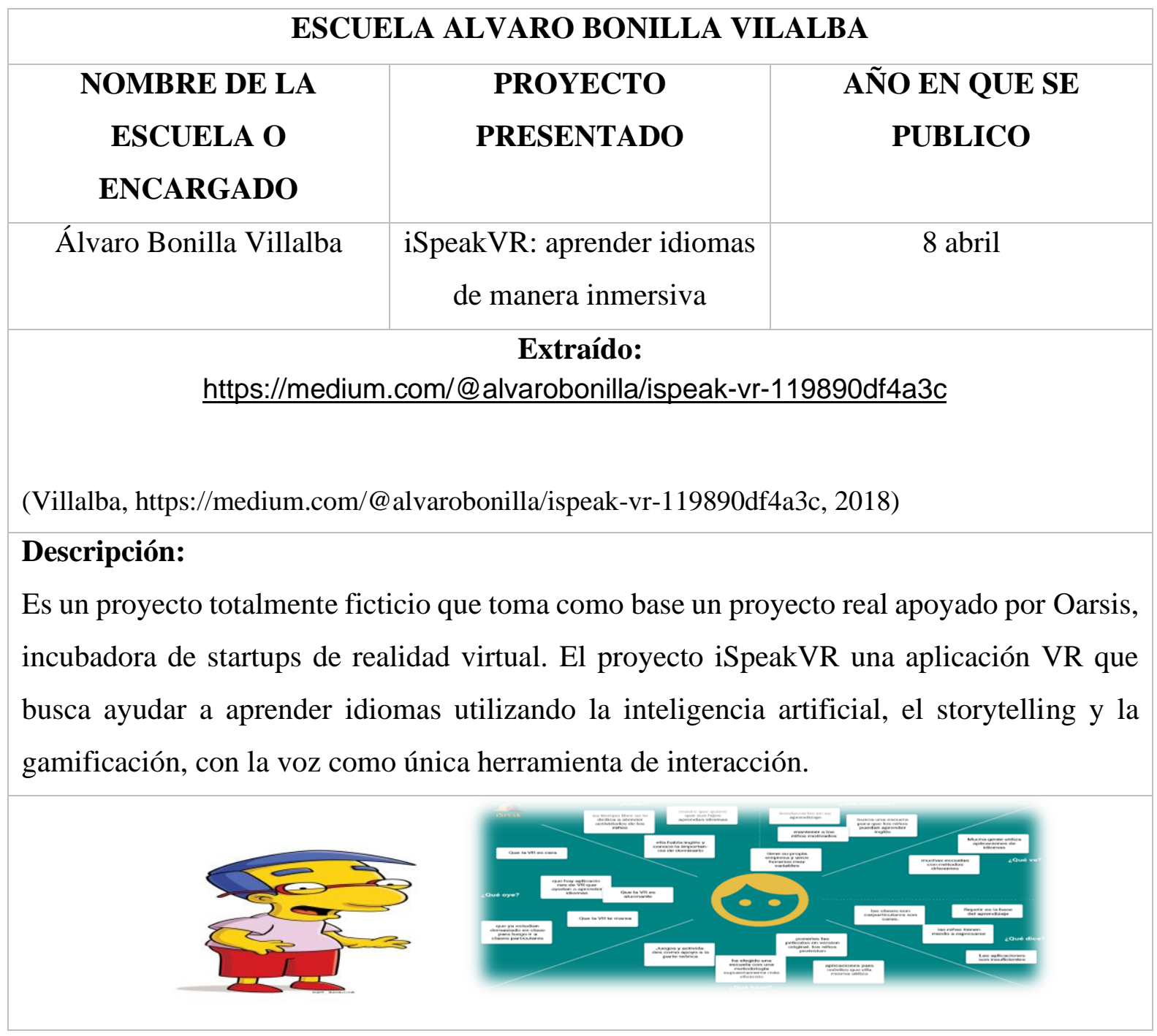

\begin{tabular}{|c|c|c|}
\hline \multicolumn{3}{|c|}{ UNIVERSIDAD AUTÓNOMA DEL ESTADO DE HIDALGO } \\
\hline $\begin{array}{c}\text { NOMBRE DE LA } \\
\text { ESCUELA O } \\
\text { ENCARGADO }\end{array}$ & $\begin{array}{c}\text { PROYECTO } \\
\text { PRESENTADO }\end{array}$ & $\begin{array}{l}\text { AÑO EN QUE SE } \\
\text { PUBLICO }\end{array}$ \\
\hline $\begin{array}{l}\text { Universidad autónoma del } \\
\text { estado de hidalgo }\end{array}$ & $\begin{array}{l}\text { Sistema de universidad } \\
\text { virtual }\end{array}$ & $\begin{array}{l}\text { Pachuca de Soto, Hidalgo, } \\
\text { Abril } 2013 .\end{array}$ \\
\hline \multicolumn{3}{|c|}{$\begin{array}{l}\text { Extraído: } \\
\text { m/bitstream/handle/123456789/14792/INGLESVII HEI } \\
2 \text { A NAVA.pdf;sequence }=2\end{array}$} \\
\hline (Olm & & \\
\hline
\end{tabular}




\section{Descripción:}

"Virtualización de la asignatura de inglés VII para fomentar la competencia de comprensión lectora de textos técnicos a través de la plataforma educativa del Instituto Tecnológico Superior del Oriente del Estado de Hidalgo, ITESA”.
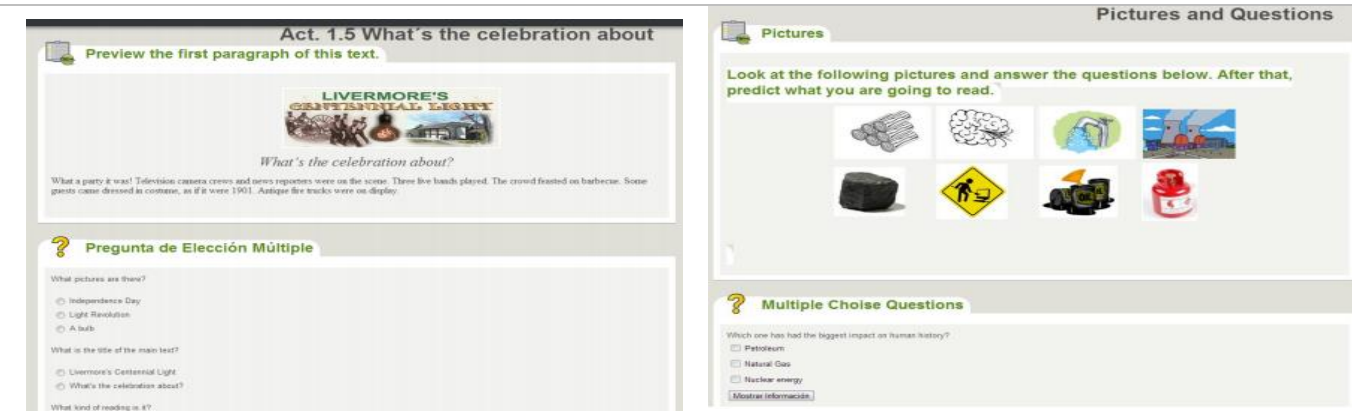

\begin{tabular}{|c|c|c|}
\hline \multicolumn{2}{|c|}{ UAEM } \\
\hline NOMBRE DE LA & PROYECTO PRESENTADO & A \\
ESCUELA O & \\
ENCARGADO & \\
\hline Universidad Autónoma del & Realidad aumentada, análisis y \\
Estado de México & aplicaciones. \\
\hline & $\begin{array}{c}\text { Extraído: } \\
\text { http://ri.uaemex.mx/handle/20.500.11799/59266 }\end{array}$ \\
\hline \multicolumn{2}{|c|}{} \\
\hline
\end{tabular}

(Arreguin Gonzalez, 2018)

\section{Descripción:}

Trabajo de investigación que pretende desglosar por completo los componentes de esta tecnología para abstraer la complejidad de la tecnología y llevarla a un plano comprensible y aplicable para las personas interesadas en el tema.

Analiza aplicaciones actuales que emplean la realidad aumentada como marco de referencia para generar nuevas aplicaciones.

NOTA: (solo es investigación). 

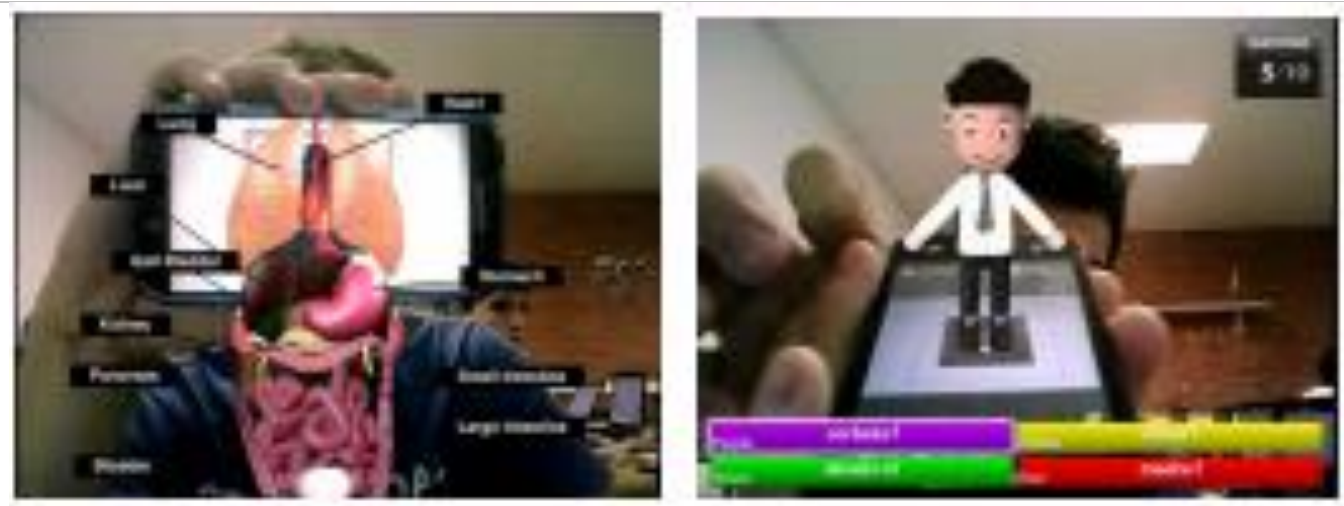

\begin{tabular}{|c|c|c|}
\hline \multicolumn{3}{|c|}{ UAEM } \\
\hline $\begin{array}{c}\text { NOMBRE DE LA } \\
\text { ESCUELA O } \\
\text { ENCARGADO }\end{array}$ & PROYECTO PRESENTADO & $\begin{array}{l}\text { AÑO EN QUE SE } \\
\text { PUBLICO }\end{array}$ \\
\hline $\begin{array}{l}\text { Escuela Superior Politécnica } \\
\text { de Chimborazo }\end{array}$ & $\begin{array}{l}\text { "Yachani Rayku Pakta" en español } \\
\text { "Aprendo por igual" }\end{array}$ & mar-2017 \\
\hline \multicolumn{3}{|c|}{$\begin{array}{c}\text { Tesis Ingeniero en Sistemas Informáticos } \\
\text { http://dspace.espoch.edu.ec/handle/123456789/6739 }\end{array}$} \\
\hline $\begin{array}{l}\text { Descripción: La aplicación c } \\
\text { español "Aprendo por igual" } \mathrm{f} \\
\text { para niños entre } 6 \text { a } 7 \text { añ } \\
\text { conjuntamente con el sdk de } \\
\text { generar un desarrollo de so } \\
\text { integración de la realidad a } \\
\text { identificaron las herramienta } \\
\text { aplicación y agregar elemento } \\
\text { de vuforia y los data sets } \mathrm{f} \\
\text { determinaron de acuerdo a la }\end{array}$ & $\begin{array}{l}\text { on realidad virtual y aumentada "Ya } \\
\text { le desarrollada como medio de aprend } \\
\text { s; esta fue creada mediante la h } \\
\text { vuforia, la librería de googleVR, y } \\
\text { tware de calidad; se analizó biblic } \\
\text { imentada y la realidad virtual en a } \\
\text { para crear el entorno de realidad au } \\
\text { propios del target (elementos multim } \\
\text { ara la aplicación; por otra parte, lo } \\
\text { iguiente clasificación: transportes, ani }\end{array}$ & $\begin{array}{l}\text { hani Rayku Pakta" en } \\
\text { aje del idioma Kichwa } \\
\text { ramienta unity } 5.4 .0 \\
\text { metodología XP para } \\
\text { rafía comprobada de } \\
\text { licativos móviles. Se } \\
\text { hentada; al elaborar la } \\
\text { dia) en la base de datos } \\
\text { entornos virtuales se } \\
\text { hales, colores, objetos; }\end{array}$ \\
\hline
\end{tabular}



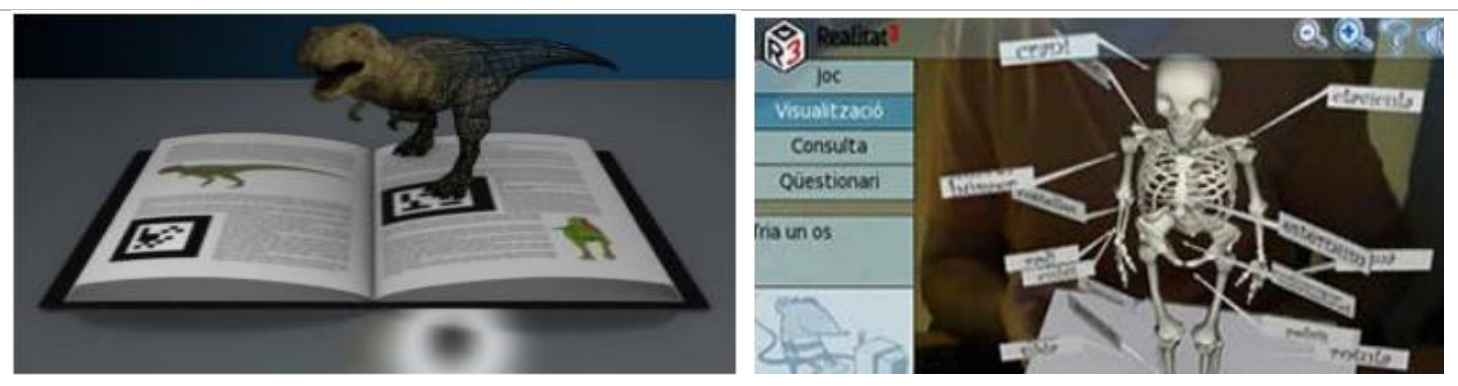

Autor: Monserrath Vargas L

TECNOLOGICO DE MONTERREY

NOMBRE DE LA PROYECTO PRESENTADO

ESCUELA O

ENCARGADO

Alencastre, Moisés; Muñoz,

Ambiente de Realidad Aumentada

Lourdes; Manrique,

Cristina; Grostieta,Zaira;

Interactivo en Tiempo Real para

2013

Rojas, Ricardo Campus

Santa Fe

Enseñar Anatomía

\section{Extraído:}

https://repositorio.itesm.mx/handle/11285/614635

https://repositorio.itesm.mx/bitstream/handle/11285/614635/NOVUS PaperAna

tomia Alencastre.pdf?sequence $=1 \&$ isAllowed $=\mathrm{y}$

$(\mathrm{Fe}, 2018)$

\section{Descripción:}

En este artículo se presenta un ambiente de Realidad Aumentada (RA) que utiliza información de una cámara de profundidad para enseñar ciertos temas de Anatomía de forma interactiva a nivel Preparatoria. La idea es mostrar a los estudiantes donde se encuentran los huesos, músculos y órganos del cuerpo humano; desplegando modelos 3D sobre el cuerpo de la persona cuya imagen está siendo capturada por un Kinect en tiempo real. De esta manera, los estudiantes pueden identificar cómo se encuentran distribuidos los huesos, 
músculos y órganos viéndose a ellos mismos como si fuera un tipo de "máquina" de rayos $\mathrm{X}$ con colores y detalles.
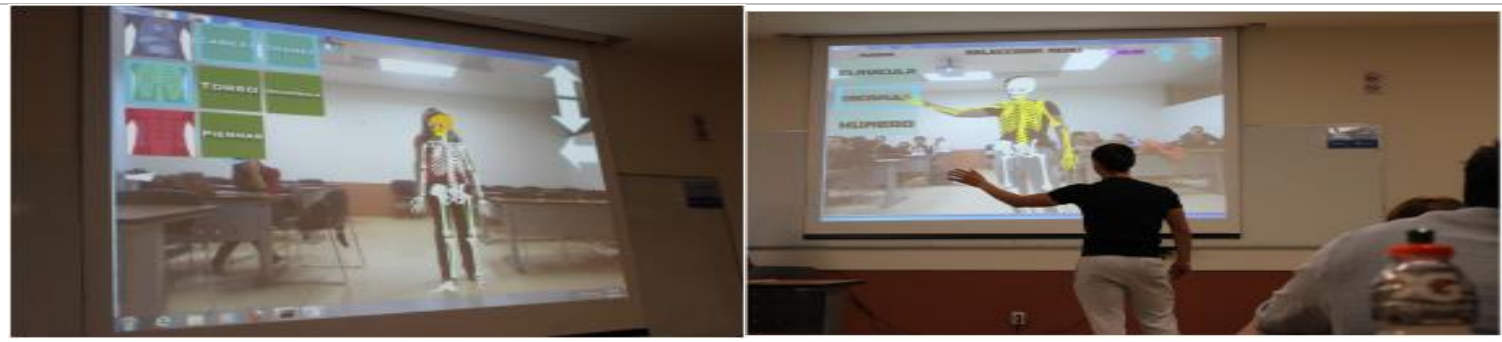

Figura 15 Estudio de Benchmarkin 